University of Nebraska - Lincoln

DigitalCommons@University of Nebraska - Lincoln

P. F. (Paul Frazer) Williams Publications

Electrical \& Computer Engineering, Department

February 1974

\title{
Resonance Fluorescence and Resonance Raman Scattering: Lifetimes in Molecular lodine
}

\author{
P. F. Williams \\ University of Nebraska - Lincoln, pfw@moi.unl.edu \\ D. L. Rousseau \\ Bell Laboratories, Murray Hill, New Jersey \\ D. H. Dworetsky \\ Bell Laboratories, Murray Hill, New Jersey
}

Follow this and additional works at: https://digitalcommons.unl.edu/elecengwilliams

Part of the Electrical and Computer Engineering Commons

Williams, P. F.; Rousseau, D. L.; and Dworetsky, D. H., "Resonance Fluorescence and Resonance Raman Scattering: Lifetimes in Molecular lodine" (1974). P. F. (Paul Frazer) Williams Publications. 34.

https://digitalcommons.unl.edu/elecengwilliams/34

This Article is brought to you for free and open access by the Electrical \& Computer Engineering, Department of at DigitalCommons@University of Nebraska - Lincoln. It has been accepted for inclusion in P. F. (Paul Frazer) Williams Publications by an authorized administrator of DigitalCommons@University of Nebraska - Lincoln. 


\title{
Resonance Fluorescence and Resonance Raman Scattering: Lifetimes in Molecular Iodine
}

\author{
P. F. Williams, D. L. Rousseau, and S. H. Dworetsky \\ Bell Laboratories, Murray Hill, New Jersey 07974
}

(Received 21 November 1973)

\begin{abstract}
We report a significantly shortened scattering lifetime in molecular iodine when the frequency of a single-mode $5145-\AA$ incident laser is tuned away from resonance with a discrete transition. This observation of a shortened scattering time is indicative of what has been conventionally called a Raman process. Off-resonance lifetime measurements, as reported here, may provide a means of determining the relative contribution to the absorption linewidth of homogeneous and inhomogeneous broadening.
\end{abstract}

We have made direct measurements of the scattering lifetime in molecular iodine as a function of the incident excitation frequency. The incident laser frequency was tuned from resonance to over $2.5 \mathrm{GHz}$ off resonance, with a transition to a discrete state below the $B\left({ }^{3} \Pi_{0+u}\right)$ dissociation limit. When the frequency was on resonance, we observed a lifetime of about $1 \mu$ sec. When the frequency was moved off resonance, the scattering time because very short, and hence "Ramanlike." Although related experiments have been reported in Mössbauer spectroscopy, ${ }^{2}$ this is the first time direct off-resonance scattering-time measurements have been made, and they show unambiguously that Raman scattering occurs under such circumstances, thereby settling a question on which there recently has been considerable speculation. ${ }^{3,4}$ The differences between the re-emission below the dissociation limit, which is strongly excitation-frequency dependent, and the well-understood scattering above the dissociation limit ${ }^{5}$ now may be interpreted properly. In addition, from off-resonance lifetime measurements a quantitative determination of the relative strengths of the homogeneous and inhomogeneous broadening should be possible.

In fluorescence, when the incident frequency coincides with a transition between a groundand an excited-state energy level, the lifetime for the re-emission process is governed by the natural lifetime of the excited state. When the incident frequency is well off resonance with the transition, the re-emission is governed by the usual properties of Raman scattering. As the incident frequency is moved only slightly away from resonance, the lifetime $(\Delta t)$ for the reemission is expected to be limited by the frequency difference $(\Delta \omega)$ between the excited state and the incident frequency, and should be given approximately by an uncertainty relationship, $\Delta t$
$=1 / \Delta \omega$. That is, if energy is not conserved, in the transition to the excited state, by an amount $\Delta \omega$, then the time the molecule can spend in the state is limited to $1 / \Delta \omega$. Since the natural fluorescence lifetimes for the $I_{2}$ discrete state ${ }^{6}$ are in the range of $10^{-6} \mathrm{sec}$, shifts in the incident laser frequency of a few gigahertz should result in dramatic changes in the scattering time.

We have made such lifetime measurements on an apparatus built and described by Bachrach ${ }^{7}$ in which the time decay is measured by a delayed-coincidence technique. The laser beam was modulated acousto-optically giving 100-nsec rectangular pulses with a $3-n s e c$ rise time. Lifetimes ranging from about a $10-$ nsec lower limit could readily be measured. The single-mode laser was tuned through the Doppler profile of the 5145- $\AA$ line, and components of the emission triplet from the $P(13)$ and $R(15)$ transitions ${ }^{8}$ were observed with a spectrometer of resolution less than $2 \mathrm{~cm}^{-1}$. All data were obtained in the frequency region of the vibrational fundamental (a frequency shift from the incident laser of about $\left.212 \mathrm{~cm}^{-1}\right)$.

In Fig. 1 we present the lifetime measurements made on the $Q$ branches resulting from the $P(13)$ and $R(15)$ excitation transitions of $\mathrm{I}_{2}$ at a pressure of $\sim 0.03$ Torr. Similar results were obtained from the $S$ branch of the $R(15)$ transition. The center of the resonance was taken to be the place where the overall fluorescence in the 200to $220-\mathrm{cm}^{-1}$ region resulting from the $R(15)$ and $P(13)$ transitions reached a maximum intensity. The $1 / e$ lifetime of the on-resonance $(0 \mathrm{GHz}$ in Fig. 1) decay of $\sim 300 \mathrm{nsec}$ is significantly shorter than that recently reported by Capelle and Broida. ${ }^{6}$ This results from the diffusion of iodine molecules out of the region focused on the spectrometer entrance slit, thereby selectively discriminating against the long-lived events. 


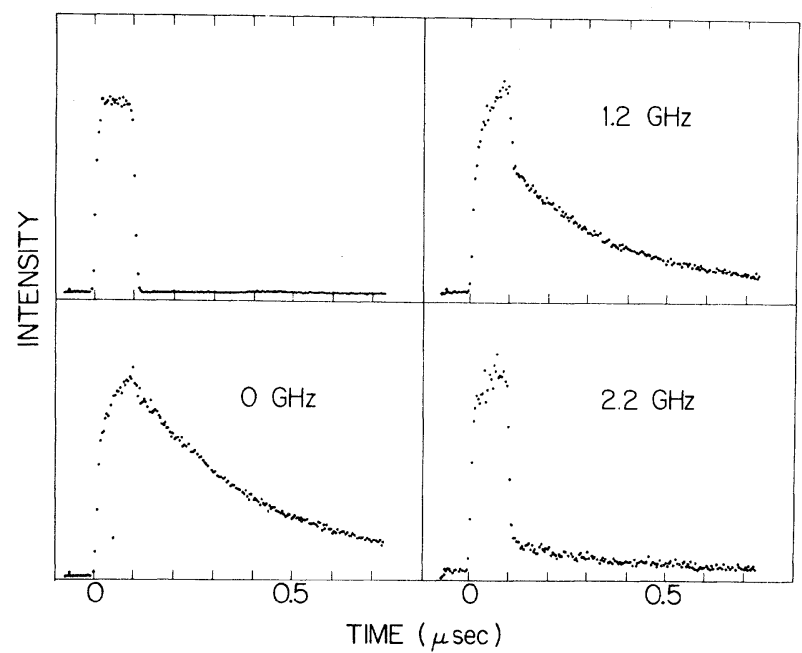

FIG. 1. Temporal response of the radiation from the $Q$ branches of the $P(13)$ and $R(15) \Delta v=1$ transitions in iodine. The various incident laser frequency shifts are relative to the fluorescence maximum. The incident laser pulse (upper left-hand corner) has a time width of $\sim 100 \mathrm{nsec}$.

With larger slits, we recorded lifetimes of $\sim 1$ $\mu$ sec, consistent with the data of Capelle and Broida. ${ }^{6}$

Off resonance, short-lifetime components appear. At $1.2 \mathrm{GHz}$, a component that follows the laser pulse in addition to the long-lived component may be seen. From $1.6 \mathrm{GHz}$ off resonance to as far as measurements were made $(2.6 \mathrm{GHz})$, the decay response did not change and was dominated by the short-lived component as exemplified by the 2.2-GHz decay curve in Fig. 1.

On the basis of the uncertainty principle and of the perturbation calculation discussed below, the transition from resonance fluorescence to resonance Raman scattering is expected to be continuous. As the laser frequency is tuned away from resonance with a discrete transition, both the re-emission intensity and lifetime must continuously decrease. Exact-resonance Raman scattering, for which $\Delta \omega=0$, and resonance fluorescence are the same long-lived process. This process is a specific case of the more general process normally called Raman scattering.

In light of the above considerations the $1.2-\mathrm{GHz}$ decay curve in Fig. 1 at first appears very surprising. A superficial analysis might lead one to believe that it indicates the presence of two distinguishable processes-short-lived resonance Raman scattering and long-lived resonance fluorescence. It must be remembered, however, that a frequency shift as small as $20 \mathrm{MHz}$ from resonance results in an uncertainty-limited lifetime of 10 nsec, the lower limit of our temporal resolution. In addition the absorption line shape of iodine is not determined by its natural (radiative and nonradiative) lifetime, but primarily by the Doppler broadening and the hyperfine splitting, ${ }^{9}$ resulting in a total linewidth of over $1 \mathrm{GHz}$. Consequently, incident frequencies in the wings of the absorption tail (see 1.2-GHz spectrum in Fig. 1) are on resonance $(\Delta \omega<100 \mathrm{kHz})$ with some components within the Doppler profile, and very far off resonance $(\Delta \omega>20 \mathrm{MHz})$ with others, giving the long- (natural lifetime) and short- (<10 nsec) lived contributions, respectively. It is easy to see that the contribution of molecules with intermediate resonant frequency shifts $(1<\Delta \omega<20 \mathrm{MHz})$ is small, and the resulting re-emission with intermediate scattering time is swamped by the other two components. These data are therefore consistent with our theoretical interpretation.

The expected time response for the re-emission process may be determined from second-order perturbation theory. Consider a three-level system consisting of ground state $|g\rangle$ with zero energy, intermediate state $|n\rangle$ with a natural linewidth $\Gamma$ and energy $\hbar \omega_{n}$, and final state $|m\rangle$ of energy $\hbar \omega_{m}$. The total observed re-emission intensity $R(t)$ then should be given by

$$
R(t) \propto(d / d t) \int|\langle m \mid \psi(t)\rangle|^{2} d \omega,
$$

where $|\psi(t)\rangle$ is the actual state of the system at time $t$ and $\omega$ is the frequency of the re-emitted light. At optical frequencies transitions from $|g\rangle$ to $|m\rangle$ do not occur so the transition must proceed via an intermediate state $|n\rangle$. We take the laser field $E_{L}$ to be turned on at $t=0$ and turned off at $t=T$, and to be given by

$$
\begin{aligned}
& E_{L}=e^{-i \omega_{L} t}\left(1-e^{-\gamma t}\right), \quad 0 \leqslant t \leqslant T, \\
& E_{L}=e^{-i \omega_{L} t}\left(1-e^{-\gamma \boldsymbol{T}}\right) e^{-\gamma(t-T)}, \quad t \geqslant T .
\end{aligned}
$$

The finite rise and fall times, with time constant $\gamma$, are assumed in order to turn the excitation on and off adiabatically. We select $\gamma$ such that $\omega_{L} \gg \gamma \gg \Gamma$. The calculation may be considerably simplified by noting that

$$
(d / d t) \int\left|\left\langle m \mid \psi_{2}(t)\right\rangle\right|^{2} d \omega \propto\left|\left\langle n \mid \psi_{1}(t)\right\rangle\right|^{2},
$$

where $\left\langle m \mid \psi_{2}(t)\right\rangle$ is derived from second-order perturbation theory and $\left\langle n \mid \psi_{1}(t)\right\rangle$ results from the first-order perturbation equation

$$
\left\langle n \mid \psi_{1}(t)\right\rangle \propto \int_{0}^{+} \exp \left[-i\left(\omega_{n}-i \Gamma / 2\right)(t-\tau)\right] E_{L}(\tau) d \tau,
$$


in which $\tau$ can be thought of as the time at which the transition from state $|g\rangle$ to state $|n\rangle$ occurs.

We have performed such a calculation ${ }^{10}$ and found that on resonance $\left(\Delta \omega=\omega_{n}-\omega_{L}=0\right)$ the re-emission intensity is proportional to $\Gamma^{-2}(1$ $\left.-e^{-\Gamma t / 2}\right)^{2}$ for $0 \leqslant t \leqslant T$, and to $\Gamma^{-2}\left(1-e^{-\Gamma T / 2}\right)^{2}$ $\times e^{-\Gamma(t-T)}$ for $t \geqslant T$, i.e., on resonance the time response is slow with time constant $\Gamma$. Off resonance $(\Delta \omega \gg \gamma)$ the result is $(\Delta \omega)^{-2}\left(1-e^{-\gamma t}\right)^{2}$ for $0 \leqslant t \leqslant T$ and $(\Delta \omega)^{-2}\left(1-e^{-\gamma T}\right)^{2} e^{-2 \gamma(t-T)}$ for $t \geqslant T$, i.e., the response appears to be instantaneous in that it follows the laser pulse. By varying $\gamma$ it may be seen that the time decay varies continuously from $\Gamma$ to an "instantaneous" value. Recently, similar conclusions were reached independently by Silverstein. ${ }^{11}$

When the incident frequency is moved away from resonance, the scattering time becomes shorter, and the re-emission is more Ramanlike. In addition the re-emission becomes much weaker. However, the spectrum remains unchanged, because only very specific initial-tointermediate-state transitions are picked out by the resonance denominator. Eventually, when the incident frequency is changed sufficiently, it is in resonance with other transitions and therefore different fluorescence spectra are observed, masking the now weakened original spectrum. In contrast, above the dissociation limit no initial vibrational-rotational state is picked out by the resonant denominator since any initial state is in resonance with a transition to some continuum state. The observed spectrum in this case is the weighted sum of lines resulting from all the vibrational-rotational levels of the ground electronic state, the weighting factor being the product of the Franck-Condon factors and population factors. ${ }^{5}$ Consequently, the observed spectrum does not have the extreme laser frequency dependence seen below the dissociation limit.

To understand clearly near-resonance light scattering it is necessary to measure directly the scattering lifetime, as reported here. The reliance of previous investigator $\mathrm{s}^{3,4}$ on quenching data to study the transition from resonance fluorescence to resonance Raman scattering has resulted in some confusion. The ambiguity inherent in the quenching data results from the different optical absorption and scattering lifetime characteristics produced by inelastic and elastic collisional broadening. As pointed out by St. Peters and Silverstein, ${ }^{12}$ the use of quenching data to measure the scattering time is valid only in the case in which elastic collisional broadening is unimportant. This is not the case for these transitions in molecular iodine, however, so direct scattering time measurements are required. We have observed that for excitation farther off resonance than $1.6 \mathrm{GHz}$, the intensity of the long-lived component ceased to decrease relative to the intensity of the short-lived component. In order to investigate this weak long-lived remnant we have made a determination of the effect increased pressure ( 0.25 Torr of $\mathrm{I}_{2}$ ) has on the lifetime response. At the higher pressure a strongly enhanced long-lived contribution was seen. We interpret the pressure dependence of the long-lived radiation as an indication that it results from elastic collision processes. At low pressure ( 0.03 Tor $r$ ) the absorption line shape far from resonance is primarily determined by the natural-lifetime Lorentzian tail. However, in the high-pressure region both homogeneous broadening (from inelastic collisions) and inhomogeneous broadening (from elastic collisions) occur. ${ }^{13}$ Off resonance a fast response is produced by the contributions to the Lorentzian tail resulting from the homogeneous broadening, but the contribution from the inhomogeneous broadening results in a long-lived component. The re-emissions resulting from both broadening processes are Lorentzian and hence the ratio of the intensities remains constant as a function of frequency shift off resonance beyond $1.6 \mathrm{GHz}$.

In regions where the contribution to the homogeneous broadening resulting from inelastic collisions is much greater than the natural-lifetime contribution, the relative contributions of inelastic and elastic collisions to the total broadening can be determined by measuring the relative intensities of the long- and short-lived components. To make a quantitative determination of this ratio, though, great care is required in the handling of effects due to saturation. In a future publication, these effects will be discussed in greater detail.

We thank R. W. Dixon and P. D. Dapkus for help with and use of their time decay apparatus. We are indebted to S. L. McCall for helpful discussions.

\footnotetext{
${ }^{1}$ Strictly speaking, when the incident laser is not exactly on resonance, the re-emission process should be referred to as near-resonant Raman scattering or near-
} 
resonance fluorescence. However, we adopt in this Letter the conventional usage [see D. G. Fouche and R. K. Chang, Phys. Rev. Lett. 29, 536 (1972); R. L. St. Peters, S. D. Silverstein, M. Lapp, and C. M. Penney, Phys. Rev. Lett. 30, 191 (1973)] in which this process is termed resonance Raman scattering.

${ }^{2}$ R. E. Holland, F. J. Lynch, G. J. Perlow, and S. S. Hanna, Phys. Rev. Lett. 4, 181 (1960); F. J. Lynch, R. E. Holland, and M. Hamermesh, Phys. Rev. 120 , 513 (1960); C. S. Wu, Y. K. Lee, N. Benczer-Koller, and P. Simms, Phys. Rev. Lett. ㅁ, 432 (1960); G. zu Putlitz, Comments At. Mol. Phys. 1, 74 (1969).

${ }^{3}$ Fouche and Chang, Ref. 1 .

${ }^{4}$ Peters et al., Ref. 1.

${ }^{5}$ P. F. Williams and D. L. Rousseau, Phys. Rev. Lett. 30, 951 (1973).

${ }^{6}$ G. A. Capelle and H. P. Broida, J. Chem. Phys. $\underline{58}$, 4212 (1973).

${ }^{7}$ R. Z. Bachrach, Rev. Sci. Instrum. 43, 734 (1972).

${ }^{8}$ T. Halldorsson and E. Menke, Z. Naturforsch. 25a, 1356 (1970).

${ }^{9}$ M. D. Levenson and A. L. Schawlow, Phys. Rev. A $\underline{6}$,
10 (1972).

${ }^{10}$ P. F. Williams, "Variation of Resonant Raman Scattering Times with Excitation Frequency" (to be published).

${ }^{11}$ S. D. Silverstein, General Electric Report No. 73CRD196, 1973 (unpublished) .

${ }^{12}$ R. L. St. Peters and S. D. Silverstein, Opt. Commun. 7, 193 (1973).

${ }^{13}$ We adopt here the standard definitions of homogeneous and inhomogeneous broadening. For homogeneous broadening the lifetime of the molecular state is limited by the broadening mechanism. Radiative broad ening is of course homogeneous, and inelastic collisions, in which the quantum state of the molecule is changed, also serve to broaden the line homogeneously On the other hand an inhomogeneously broadened line is one in which the center frequency of the oscillators is distributed over a line profile, and the lifetime is the same throughout. Doppler broadening is inhomogeneous and elastic collisions, in which the phase of the oscillator is interrupted but the quantum state is unchanged, also result in inhomogeneous broadening. 